\title{
Infiltración epidural de corticosteroides. ¿Sí o no? A propósito de una alerta
}

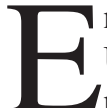
n abril de este año, la Administración de Alimentos y Medicamentos (FDA) de los Estados Unidos de Norteamérica emite un anuncio de seguridad para advertir sobre los problemas neurológicos graves, pero poco frecuentes, tras una inyección epidural de corticoides para aliviar el dolor (1).

Cabe destacar de este comunicado:

- Que se está solicitando que se añada una "advertencia" en el etiquetado de los corticoides inyectables donde se deben describir dichos riesgos (pérdida de la visión, derrame cerebral, parálisis y muerte).

- Que los pacientes deben conversar con los profesionales de la salud sobre los beneficios y riesgos de estas inyecciones como de las otras alternativas de tratamiento.

- Continúa diciendo que inyectar corticosteroides en el espacio epidural para reducir la inflamación ha sido una práctica común por muchas décadas, sin embargo, no se ha establecido la eficacia y seguridad de los medicamentos para este uso. Que la FDA no ha aprobado el uso de corticoides para esta modalidad de tratamiento y que empezó a investigar este asunto basado en un artículo publicado en el año 2009. Esta información motivó a examinar los casos que se encontraban en la base de datos del Sistema de Informes de Eventos Adversos (FAERS) de la FDA y de la literatura médica disponible (14 referencias).

- Se informa en el mismo que, con la finalidad de generar conciencia en la comunidad médica sobre el riesgo de este tipo de tratamiento, se reunió a un panel de expertos para definir las técnicas a usar en dichas inyecciones a fin de reducir el daño previsible, las cuales se darían a conocer cuando estuvieran listas. Además, se convocaría una reunión del comité asesor de expertos externos a finales de 2014 para conversar y determinar si la FDA debe tomar medidas adicionales sobre estas inyecciones.

Incluyen a todos los corticosteroides que se utilizan en la práctica diaria en este tipo de procedimiento y puntualizan que la alerta no está relacionada con la contaminación de estos productos dada a conocer en 2012.

El comunicado continúa describiendo los datos sobre los corticoides e información adicional para pacientes y profesionales de la salud donde repiten lo anteriormente mencionado.

En agosto de este mismo año, la International Spine Intervention Society (ISIS) emite una respuesta sobre este anuncio de la FDA (http://www.spinalinjection.org/): "la ISIS considera que el mensaje de alerta sobre la seguridad de las inyecciones epidurales de corticoides es engañoso y contiene información inexacta sobre la eficacia de este procedimiento". Además relata que: "la declaración fue emitida por la división reguladora de la FDA y no por la Iniciativa de Uso Seguro (SUI) de esta agencia reguladora. La FDA SUI ha estado trabajando con los médicos especialistas de varias organizaciones, entre ellas varios dirigentes de la ISIS, en los últimos dos años, con el objetivo de ayudar a crear un conjunto de recomendaciones para la administración segura de las inyecciones epidurales de esteroides. Es importante tener en cuenta que la liberación de la declaración de abril de este año por el poder regulatorio de la FDA fue unilateral y sin el 
conocimiento del grupo de expertos que trabajan con el SUI en el tema de las inyecciones epidurales de corticoides. Tras la publicación de esta declaración, el grupo de expertos implicados en este proyecto decidieron involucrar a 14 sociedades científicas representadas en el Multi-Pain Society Workgroup (MPW) con la finalidad de elaborar un conjunto de recomendaciones para la administración segura de las inyecciones epidurales de corticoides.

Dentro de este comunicado se emiten una serie de recomendaciones elaboradas por consenso del MPW con excepción de la American Society of Interventional Pain Physicians (ASIPP) que declinó su participación.

Cabe destacar que en la misma página web de la ISIS, en la sección de noticias, se publica una misiva, firmada por su presidente, donde se resalta la falta de veracidad de la alerta de la FDA en relación a la inexistencia de evidencia sobre la efectividad de las inyecciones de esteroides, alegando que existe demostrada y sobrada información sobre la eficacia del uso de corticoides en este tipo de intervenciones, no sólo reduciendo o eliminando el dolor y disminuyendo el uso de opioides, sino también, evitando en muchos pacientes el tratamiento quirúrgico.

Por su parte, La Junta Directiva de la Sociedad Española del Dolor (SED) emite un comunicado a sus socios sobre la alerta en cuestión, describiendo los puntos más importantes del mismo y emitiendo una serie de consideraciones al respecto:

1. El informe no distingue la vía de administración de corticoides, si es interlaminar o transforaminal.

2. El informe no distingue el tipo de corticoide, si es particulado o no, con las importantes consideraciones entre unos y otros.

3. Que algunas sociedades científicas estadounidenses relacionadas con el tratamiento del dolor han calificado este comunicado como inexacto y con conclusiones que pueden inducir a error, sobre todo el párrafo referente a la seguridad y la eficacia de los corticoides por vía epidural (Update on ISIS Response to FDA Statement on ESIs, June 2014).

4. La alerta de la FDA ha sido redactada de manera unilateral por la denominada "regulatory division de la FDA" y no con la "Safe Use Initiative" (SUI) de la FDA que venía trabajando en el uso de corticoides epidurales durante meses junto con un amplio panel de expertos de diversas sociedades científicas americanas dedicadas al tratamiento del dolor (MultiSociety Pain Workgroup).

Continúa el comunicado de la SED mencionado un artículo publicado en el New England Journal of Medicine (4) sobre la eficacia y seguridad de los corticoides en el tratamiento del canal estrecho, donde se concluye que: el uso de estos medicamentos combinados con lidocaína por vía epidural tienen poco o ningún beneficio en el tratamiento del dolor ocasionado por la estenosis de canal lumbar cuando se compara con la inyección sólo de lidocaína. Del mismo modo, la SED recomienda que la lectura del mismo, debido a los fallos metodológicos que presenta este estudio, se haga conjuntamente con un artículo publicado al respecto por el Dr. Manchikanti y cols. (5).

Además, dice la SED, se deben tener en cuenta las indicaciones mencionadas en la ficha técnica de estos medicamentos, sobre todo las de la triamcinolona, donde explícitamente se expone que la misma no debe utilizarse por la vía epidural.

Concluye la SED que se debe informar a los pacientes de los posibles, pero poco probables, riesgos de la administración de corticoides epidurales, modificando el consentimiento informado de las técnicas que lo incluyan. Posiblemente sea recomendable añadir un consentimiento explícito como tratamiento compasivo de los corticoides teniendo como referencia el Real Decreto que regula el uso de fármacos en condiciones especiales (6).

Termina el comunicado diciendo que en relación a este tema se ha hecho un primer contacto con la Agencia Española del Medicamento y que mantendrán informados a sus socios.

En la revista Pain Physician de julio/agosto de 2014 aparece un artículo de Manchikanti y cols. titulado "Epidural steroid warning controversy still dogging FDA" (7) donde exponen, después de estudiar detalladamente la bibliografía aportada por la FDA para soportar la alerta, las imprecisiones de la misma. Este trabajo, objetivo, meticuloso y perfectamente diagramado no deja ningún tipo de margen para discrepar y poner en evidencia que la FDA y sus consultores no revisaron, o lo hicieron de forma inadecuada, toda la literatura aportada, llevando o conduciendo como consecuencia a tomar conclusiones inadecuadas. 
Concluyen los autores que la FDA debería modificar su declaración reemplazándola con una advertencia basada en la evidencia existente, haciendo hincapié en que la indicación no oficial (off label) del uso de los corticoides por vía epidural rara vez puede ocasionar graves problemas neurológicos después de una inyección epidural transforaminal cervical o torácica, y que también se puede aumentar el riesgo de generar estos daños en inyecciones epidurales transforaminales lumbares cuando se realizan sin las precauciones adecuadas. Agregan que la conclusión de la FDA fue basada en la falta de evidencia y que, en definitiva, dificultará a los pacientes tener acceso a los tratamientos necesarios en el manejo del dolor espinal crónico.

En el número correspondiente a septiembre/octubre de la revista Pain Physician, Manchikanti y cols. (8) insisten en clarificar el tema de la alerta de la FDA entrando en clara discrepancia, y con el soporte de una carta firmada por 1.040 especialistas norteamericanos en el manejo intervencionista del dolor, ahora no sólo en contra del etiquetado propuesto de la FDA, sino también con las recomendaciones consensuadas por el MPW. Los autores de este artículo argumentan, punto a punto, la falta de evidencia de estas recomendaciones.

En los comentarios, además de una clara confrontación entre sociedades y agrupaciones norteamericanas especializadas en el manejo del dolor, critican que: tanto la Asociación Americana de Anestesiología (ASA) como la ISIS continúan argumentando que las normas de seguridad son esenciales, sin embargo, no solicitan la eliminación de la declaración de la FDA del 23 de abril de 2014, reconociendo por lo tanto, de manera implícita, que estas recomendaciones son parte de las ya emitidas por la alerta. También afirman estas sociedades que la evidencia disponible fue tomada en cuenta y que se obtuvo un consenso entre todos los interesados. Aclaran los autores de este artículo que las Sociedades agrupadas en la MPW (principalmente la ISIS y la ASA), no están dispuestas a compartir o debatir cualquier diferencia en relación a las evidencias aportadas e incluso en los principios aplicados para llegar a un consenso.

Concluye este artículo diciendo que los autores de este manuscrito y los signatarios de la carta enviada a la FDA, en relación a las recomendaciones anteriores hechas por ellos a la FDA con respecto a la alerta y al etiquetado que debe llevar la medicación corticosteroide cuando es usada por vía espinal, debe ser, en definitiva, una advertencia que haga hincapié en que el uso off-label de la infiltración epidural de corticoides puede causar problemas neurológicos raros, pero graves, después de una inyección epidural transforaminal cervical o torácica y, tal vez, puede estar asociada con un aumento de estos riesgos en la inyección epidural lumbar transforaminal cuando esta se realiza sin las medidas correctas. Además puntualizan que todos los procedimientos deben ser realizados por personas bien entrenadas y en establecimientos apropiados que dispongan de fluoroscopia u otras modalidades idóneas de imágenes.

En el intervalo de tiempo mientras redactamos este artículo han ido apareciendo nuevas referencias que creemos deben ser revisadas por los interesados en este asunto $(9,10)$.

Nosotros, como médicos especialistas en dolor intervencionista, nos vemos en la obligación y en la imperiosa necesidad de emitir nuestra opinión en este tema. Alrededor del $70 \%$ de los procedimientos que realizamos en nuestra clínica son infiltraciones epidurales con medicación corticosteroide, por consiguiente, nos preocupan las influencias legales que este comunicado de la FDA pueda tener. Se han dado siempre como norma a nivel mundial las decisiones tomadas por esta Agencia Gubernamental de EE. UU. Creemos que en esta cuestión no sólo se ha equivocado sino que lo ha hecho de manera irresponsable. También creemos que la respuesta por las organizaciones y sociedades especializadas en la materia, con algunas excepciones, ha sido poco contundente y consensuada y pasamos a argumentar el porqué en orden cronológico:

1. No entendemos de qué sirve una advertencia en un etiquetado cuando una medicación no está expresamente autorizada para un uso específico. Creemos que lo correcto sería aprobar su uso con una etiqueta advirtiendo los posibles riesgos que se pudieran suceder.

2. Pareciera que la FDA desconociera el modo de funcionamiento de las Clínicas del Dolor. Aproximadamente el $90 \%$ de nuestros pacientes nos son referidos de otras especialidades cuando fallan los tratamientos de primera línea (fármacos, rehabilitación física, osteopatía, terapias complementarias, etc.) o cuando los cirujanos especializados en este tipo de patología prefieren intentar, como opción previa al tratamiento quirúrgico, una infiltración epidural para decidir la conducta a seguir. Esto quiere decir, siguiendo los lineamientos de 
la FDA, que la discusión dentro de las alternativas de tratamiento estaría entre no hacer nada o la cirugía. La opción quirúrgica en este tipo de patología no sólo es limitada en cuanto a buenos resultados se refiere, sino que además existe mayor probabilidad de causar los daños descritos en la alerta en cuestión (11-22).

Es difícil entender que la indicación y utilización de estos medicamentos por esta vía "que es una práctica común y por décadas" no haya sido discutida por esta Agencia con anterioridad. Además, afirma que "carecen de eficacia y seguridad" sin los correspondientes estudios comprobatorios que demuestran lo contrario.

3. Las 14 referencias que soportan esta alerta, encontradas en un periodo de tiempo entre el 2003 al 2010, son casos clínicos reportados relacionados con inyecciones de corticoides vía epidural, encontrándose los mismos en el nivel más bajo de cualquiera de las escalas de la Medicina Basada en la Evidencia. Además, se incluyen en estas, no sólo las graves lesiones que pudieran ocasionar el embolismo de corticoides, sino también: lesiones y espasmos vasculares, complicaciones evidentes de la técnica operatoria, embolismo por arterias diferentes a las arterias radiculares, comorbilidades y un caso sin aparente explicación. Creemos difícil afirmar que el uso de estos medicamentos, basados en la información descrita, es potencialmente peligroso. Pero no sólo esto, la referencia 17 (23) de esta comunicación no tiene nada que ver con el tema que nos preocupa, sólo trata sobre la seguridad de la técnica del bloqueo epidural en todos los ámbitos de su uso. Cabe destacar que su uso en dolor crónico muestra, en el enfoque optimista, con 0 complicaciones fatales.

4. No se justifica la inclusión de todas las formulaciones corticosteroideas cuando en ninguno de los casos aportados como referencia se utilizó la presentación no particulada de estos (dexametasona).

Con respecto al comunicado de la ISIS en conjunto con la MWP, coincidiendo con el grupo de Manchikanti, creemos conveniente que el mismo debería enfocarse, además de establecer algunos estándares para la práctica segura de la técnica, hacia la exigencia de solicitar la aprobación por parte de la FDA de que el uso y etiquetado de los corticoides muestren, por lo menos hasta que se demuestre de forma evidente lo contrario, que pueden ser utilizados por la vía epidural con la advertencia sugerida por este mismo grupo y por 1.040 especialistas del manejo intervencionista del dolor.

En cuanto a las recomendaciones que se han propuesto por parte de la MWP, además de las precisas observaciones llevadas a cabo por Manchikanti y cols., creemos conveniente contraindicar el uso de la inyección transforaminal cervical, o por lo menos, limitarlo a casos muy especiales, sobre todo en niveles como $\mathrm{C} 1 / \mathrm{C} 2$ y C7/T1, donde la arteria vertebral se expone al dejar su protección ósea y donde sus variables anatómicas se observan con mayor frecuencia.

Compartimos el comunicado de la Sociedad Española del Dolor (SED) aunque excluiríamos del mismo el ensayo clínico anexado a este.

El trabajo publicado en el New England Journal of Medicine titulado "A randomized trial of epidural glucocorticoid injections for spinal stenosis" (4), y que fue a la vez acompañado de un editorial en el mismo número de la revista, fue duramente criticado por Manchikanti y cols. (6) debido a las severas limitaciones del estudio, del manuscrito y del editorial que lo acompaña. El diseño, criterios de inclusión, evaluación de resultados, análisis e interpretación de datos y las conclusiones de este muy "publicitado, randomizado y altamente sofisticado estudio" no son apropiadas y conllevan a la desinformación.

Además de todo lo descrito en este minucioso análisis, bastaría solo con mirar la tabla 3 del estudio "Eventos adversos y supresión del cortisol a las 6 semanas" donde incluyen a la punción dural dentro de este renglón cuando no sólo debe ser excluido de los grupos en estudio (n) por ser una complicación de la técnica, sino que estos pacientes no deberían haber recibido ningún tipo de medicación.

Estamos completamente de acuerdo con los criterios, observaciones y proposiciones realizadas por Manchikanti y cols. y la reacción de los 1.040 especialistas en dolor intervencionista de EE. UU. expresadas en los artículos previamente mencionados. Creemos que esta es la vía, sino la única, de argumentar y proponer soluciones a temas tan delicados como emitir una alerta de este tipo y por una Agencia Gubernamental tan influyente. El previo estudio serio, minucioso y la consecuente discusión, con criterios de inclusión, con los organismos reguladores, sociedades 
científicas, e inclusive con particulares interesados, debe ser siempre la forma correcta de encontrar un resultado consensuado.

Como conclusión comentar que en los EE. UU. se realizaron 2.289.213 infiltraciones epidurales tan sólo en su servicio de seguridad social (Medicare and Medicaid) en el año 2011 (24), en comparación a la poca o escasa evidencia aportada por la FDA, como para generar una alarma a un procedimiento tan antiguo, común y efectivo. No dudamos que haya más casos con severas complicaciones sin reportar debido a los problemas legales que esto pueda suponer o por lo difícil de realizar un estudio serio con las variables necesarias, pero la evidencia no contempla suposiciones. No queremos pensar que esta advertencia tenga alguna relación con algún beneficio de parte de la industria relacionada a este tipo de actividad.

Creemos que toda alerta es necesaria, siempre y cuando esté bien documentada, de lo contrario lo que genera es pánico y su consecuente limitación en cuanto a las opciones de tratamiento disponibles.

Decimos necesaria porque por lo menos en este caso nos obliga de alguna forma a considerar, evaluar e informar a nuestros pacientes más exhaustivamente al momento de ofrecerles este tipo de intervenciones. También hemos notado que las últimas generaciones de médicos intervencionistas en dolor, posiblemente debido al desarrollo de técnicas de imágenes como la ecografía, le concedan más importancia a la posible lesión o trauma nervioso, minimizando las complicaciones que se puedan generar sobre las estructuras vasculares y el consecuente infarto medular o cerebral.

Podemos observar, en la escasa bibliografía aportada por la FDA para soportar esta alerta, que la isquemia de las estructuras nerviosas no sólo se puede generar por embolismos del componente particulado de una medicación, sino también por trauma o lesión directa, compresiones extrínsecas (hematomas o abscesos), espasmo o por alguna enfermedad desconocida por el paciente que pueda ocasionar, de alguna forma, una predisposición a sufrir este tipo de complicación vascular.

Nosotros y un grupo de médicos intervencionistas que hemos consultado que trabajan en España y fuera de ella seguimos realizando este tipo de intervenciones, con el agravante de que la balanza sobre el riesgo de su utilización se inclina, por más explicaciones y consentimientos informados expeditos que se obtengan, a favor de los practicantes de esta técnica y no del enfermo, como debería ser. No es completamente cierto que debido a que nuestra Agencia de Medicamentos no se haga eco de esta alerta nos protege de alguna forma en caso de algún accidente. La globalización de la información no tiene barreras y menos aún en casos de un resultado dramático.

Urge acordar un amplio consenso para llegar de alguna forma a justificar que lo que hacemos es lo adecuado y lo correcto.

\author{
C. L. Nebreda, A. Ojeda y L. Aliaga
}

Clínica del Dolor. Hospital Quirón Teknon. Barcelona

\title{
BIBLIOGRAFÍA
}

1. http://www.fda.gov/Drugs/DrugSafety/ucm394280

2. http://www.spinalinjection.org/

3. portal.sedolor.es

4. Friedly JL, Comstock BA, Turner JA, Heagerty PJ, Deyo RA, Sullivan SD, et al. A randomized trial of epidural glucocorticoid injections for spinal stenosis. N Engl J Med 2014;371:11-21.

5. Manchikanti L, Candido KD, Kaye AD, Boswell MV, Benyamin RM, Falco FJE, et al. Randomized trial of epidural injections for spinal stenosis published in The New England Journal of Medicine: Further confusion without clarification. Pain Physician 2014;17:E475-E487.

6. http://www.boe.es/boe/dias/2009/07/20/pdfs/BOE-A-200912002.pdf.

7. Manchikanti L, Candido KD, Singh V, Gharibo CG, Boswell MV, Benyamin RM, Epidural steroid warning controversy still dogging FDA. Pain Physician 2014;17:E451-E474.

8. Manchikanti L, Falco FJE, Benyamin RM, Gharibo CG, Candido KD, Hirsch JA. Epidural steroid injections safety recommendations by the Multi-Society Pain Workgroup (MPW): More regulations without evidence or clarification. Pain Physician 2014;17:E575-E588. 
9. Huntoon MA, Buvanendran A. Transforaminal epidural steroid injections anatomical and imaging analysis trumps other agendas. Regional Anesthesia and Pain Medicine 2014;39(6).

10. Nishio I. Cervical transforaminal epidural steroid injections. A proposal for optimizing the preprocedural evaluation with available imaging. Regional Anesthesia and Pain Medicine 2014;39(6).

11. Klezl Z, Swamy GN, Vyskocil T, Kryl J, Stulik J. Incidence of vascular complications arising from anterior spinal surgery in the thoraco-lumbar spine. Asian Spine J 2014;8(1):59-63.

12. Marquez-Lara A, Nandyala SV, Hassanzadeh H, Noureldin M, Sankaranarayanan S, Singh K. Sentinel events in cervical spine surgery. Spine (Phila Pa 1976) 2014;39(9):715-20.

13. Lunardini DJ, Eskander MS, Even JL, Dunlap JT, Chen AF, Lee JY, et al. Vertebral artery injuries in cervical spine surgery. Spine J 2014;14(8):1520-5.

14. Cox JB, Weaver KJ, Neal DW, Jacob RP, Hoh DJ. Decreased incidence of venous thromboembolism after spine surgery with early multimodal prophylaxis. J Neurosurg Spine 2014;21(4):677-84.

15. Grimm BD, Leas DP, Poletti SC, Johnson DR 2nd. Postoperative complications within the first year after extreme lateral interbody fusion: Experience of the first 108 patients. J Spinal Disord Tech 2014.

16. Marquez-Lara A, Nandyala SV, Fineberg SJ, Singh K. Cerebral vascular accidents after lumbar spine fusion. Spine (Phila Pa 1976) 2014;39(8):673-7.

17. Fineberg SJ, Ahmadinia K, Patel AA, Oglesby M, Singh K. Incidence and mortality of cardiac events in lumbar spine surgery. Spine (Phila Pa 1976) 2013;38(16):1422-9.

18. Goz V, Weinreb JH, McCarthy I, Schwab F, Lafage V, Errico TJ. Perioperative complications and mortality after spinal fusions: Analysis of trends and risk factors. Spine (Phila Pa 1976) 2013; 38(22):1970-6.

19. Ong KL, Auerbach JD, Lau E, Schmier J, Ochoa JA. Perioperative outcomes, complications, and costs associated with lumbar spinal fusion in older patients with spinal stenosis and spondylolisthesis. Spine J 2013. pii: S1529-9430(13).

20. Goldstein CL, Bains I, Hurlbert RJ .Symptomatic spinal epidural hematoma after posterior cervical surgery: Incidence and risk factors. Spine J 2013. pii: S1529-9430(13).

21. Mannion AF, Fekete TF, O'Riordan D, Porchet F, MutterU M, Jeszenszky D, et al. The assessment of complications after spine surgery: Time for a paradigm shift? Spine J 2013;13(6):615-24.

22. Yoshihara H, Yoneoka D. Incidental dural tear in lumbar spinal decompression and discectomy: Analysis of a nationwide database. Arch Orthop Trauma Surg 2013;133(11):1501-8.

23. Cook TM, Counsell D, Wildsmith JA; Royal College of Anaesthetists Third National Audit Project. Major complications of central neuraxial block: Report on the Third National Audit Project of the Royal College of Anaesthetists. Br J Anaesth 2009;102:179-90.

24. Manchikanti L, Pampati V, Falco FJ, Hirsch JA. Assessment of the growth of epidural injections in the Medicare population from 2000 to 2011. Pain Physician 2013;16(4):E349-E364. 